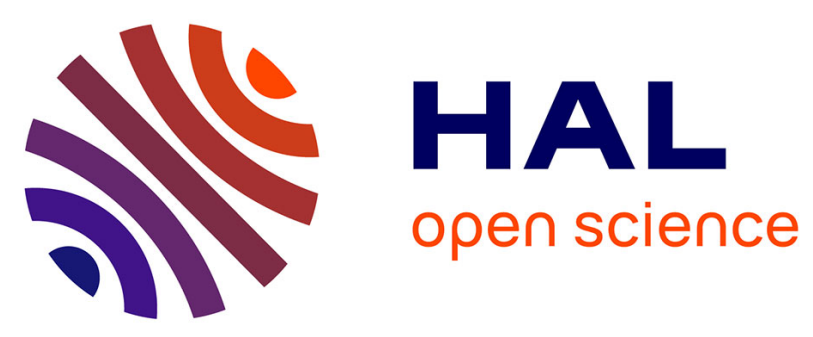

\title{
Laminar flow friction factor in highly curved helical pipes: Numerical investigation, predictive correlation and experimental validation using a 3D-printed model
}

Omran Abushammala, Rainier Hreiz, Cécile Lemaitre, Éric Favre

\section{- To cite this version:}

Omran Abushammala, Rainier Hreiz, Cécile Lemaitre, Éric Favre. Laminar flow friction factor in highly curved helical pipes: Numerical investigation, predictive correlation and experimental validation using a 3D-printed model. Chemical Engineering Science, 2019, 207, pp.1030-1039. 10.1016/j.ces.2019.07.018 . hal-02197694

\section{HAL Id: hal-02197694 \\ https://hal.univ-lorraine.fr/hal-02197694}

Submitted on 25 Oct 2021

HAL is a multi-disciplinary open access archive for the deposit and dissemination of scientific research documents, whether they are published or not. The documents may come from teaching and research institutions in France or abroad, or from public or private research centers.
L'archive ouverte pluridisciplinaire HAL, est destinée au dépôt et à la diffusion de documents scientifiques de niveau recherche, publiés ou non, émanant des établissements d'enseignement et de recherche français ou étrangers, des laboratoires publics ou privés.

\section{(ㄷ)(1) $\$$}

Distributed under a Creative Commons Attribution - NonCommerciall 4.0 International 


\title{
1 Laminar flow friction factor in highly curved helical pipes: 2 numerical investigation, predictive correlation and experimental validation using a 3D-printed model
}

\author{
5 Omran Abushammala, Rainier Hreiz*, Cécile Lemaître, Éric Favre \\ Laboratoire Réactions et Génie des Procédés, Université de Lorraine, CNRS, LRGP, F-54000 Nancy, France. \\ "Corresponding author at: \\ Rainier Hreiz, Laboratoire Réactions et Génie des Procédés, Université de Lorraine, ENSIC, CNRS, LRGP, 1 rue Grandville, 54001 Nancy, \\ France. Tel: +33 (0) 372743 876; E-mail address: rainier.hreiz@univ-lorraine.fr
}

\begin{abstract}
Highly curved helical pipes offer attracting potentialities for intensified mass/heat transfer performances as they generate intense Dean-type vortices. The evaluation of friction factor in such geometries is necessary for assessing the trade-offs between the increase of transfer efficiency and the associated specific energy requirement. Unfortunately, such data are lacking for highly curved helixes, probably due to the difficulty to manufacture these geometries through traditional manufacturing techniques.

In this paper, CFD simulations are carried out for determining the laminar flow friction factor in helical pipes, particularly highly curved ones. For an experimental validation of the numerical results, a highly curved helix was built by 3D-printing. Existing correlations are shown to fail for the accurate prediction of the friction factor in highly curved helixes. A new correlation is thus proposed. An excellent agreement is obtained between the experimental pressure drop measurements and the proposed correlation predictions.
\end{abstract}

Keywords: 3D printing; CFD; Correlation; Dean vortices; Friction factor; Helical pipe

\section{Introduction}

Hydrodynamics in curved pipes is characterized by the occurrence of secondary flows, i.e. recirculations of relatively low magnitude in the cross-stream direction, perpendicularly to the primary flow. In toroidal pipes and bends, they consist of a symmetric pair of counter-rotating vortices known as Dean cells, Fig. 1a, after Dean (1927, 1928). In these pioneering papers, Dean derived an analytical solution for the laminar flow hydrodynamics in toroidal pipes which curvature radius is much larger than the pipe diameter. The so-called Dean number, $D e$, is generally used to quantify the intensity of these secondary flows:

$$
D e=\operatorname{Re} \sqrt{\frac{d}{2 R}}=\frac{\rho d U}{\mu} \sqrt{\frac{d}{2 R}}
$$

where $R e$ represents the Reynolds number, $\rho$ the fluid density, $\mu$ the fluid dynamic viscosity, $U$ the mean velocity of the primary flow, $d$ the internal diameter of the pipe and $R$ the radius of the torus/bend. This dimensionless number is often used as the sole representative parameter in correlations concerning the flow characteristics and the heat and mass transfer efficiencies in curved pipes; indeed, the magnitude of these transport phenomena is strongly correlated to the intensity of Dean vortices.

(a)
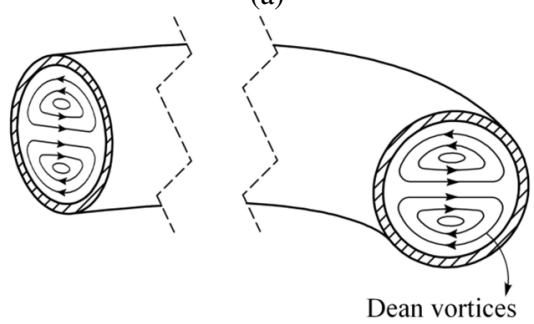

(b)

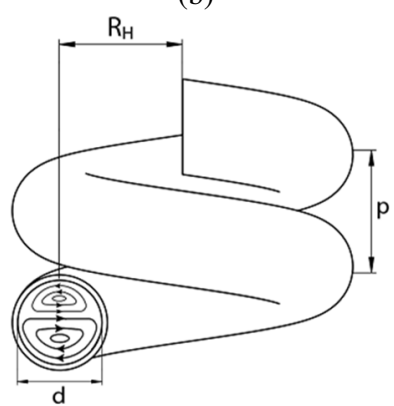

Figure 1: Schematic representation of Dean cells: (a) in a bend or a toroidal pipe. (b) in a helical tube.

In the case of helical pipes, Dean-type cells still develop, however, their symmetry is broken because of torsion effects (Figure 1b). This geometry is widely used in industrial processes and applications where the presence of secondary flows allows reaching much better performance than straight pipes, e.g.: (1) Heat transfer 
enhancement. Helically coiled heat exchangers are commonly encountered in anaerobic digesters (Liu et al., 2019), refrigeration systems (Gill and Singh, 2018), power plants and nuclear reactors (Pioro, 2016) among many other applications. (2) Mass transfer enhancement in catalytic reactors (Abdel-Aziz et al., 2010), dense (Mendez et al., 2017) and porous (Ghidossi et al., 2006) membrane contactors, etc. (3) Fouling and clogging reduction in filtration membranes (Moll et al., 2007). (4) Improvement of mixing efficiency and homogenization (Mansour et al., 2017).

Many studies focusing on flow and/or transport phenomena in helical pipes relied upon the Dean number as the sole dimensionless parameter accounting for both flow and geometry effects (see Table 1 for example). Most of these authors extended the Dean number definition to helical geometries simply by replacing the pipe curvature radius with the helix radius, $R_{H}$ (Figure 1b), in the original form (Eq. 1):

$$
D e_{R_{H}}=\frac{\rho d U}{\mu} \sqrt{\frac{d}{2 R_{H}}}=R e \sqrt{\frac{d}{2 R_{H}}}
$$

However, doing so, the effects of the helix pitch are completely ignored, which may lead to important errors in several situations. Effectively, as discussed later in this paper, the flow characteristics may substantially vary with the helix pitch, especially in the case of pipes with a small helix radius.

Table 1: Set of commonly used correlations for predicting the friction factor of fully developed laminar flow in helical pipes. $C_{f, H}^{\infty}$ and $C_{f, S}^{\infty}$ correspond to the asymptotic friction factor in helical and straight pipes respectively. $C_{f, S}^{\infty}$ is equal to 64/Re following the Darcy-Weisbach definition which is used in this paper.

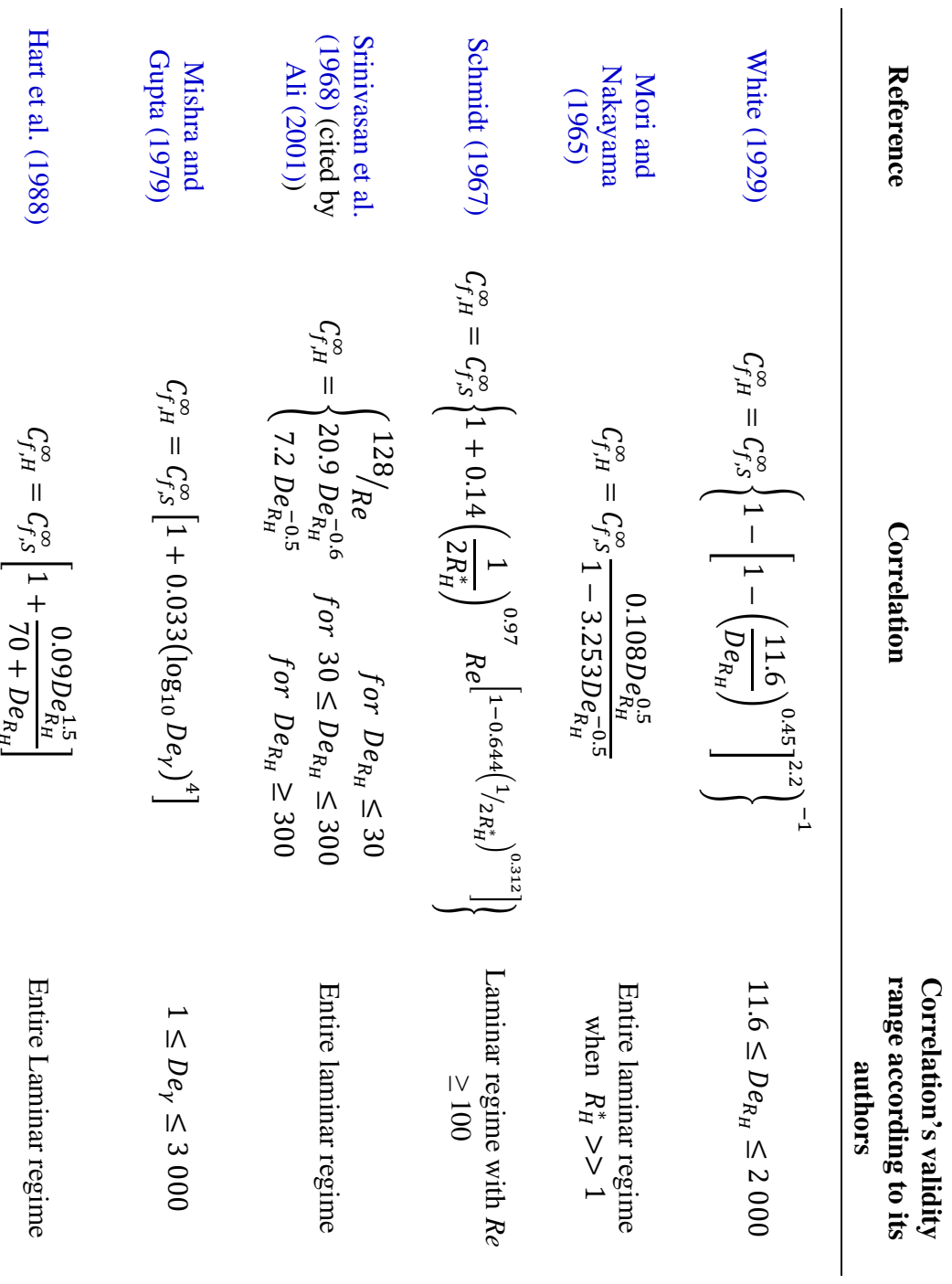


On the other hand, some authors adopted a different expression for the Dean number in helical pipes as follows:

$$
D e_{\gamma}=\frac{\rho d U}{\mu} \sqrt{\frac{d}{2 \gamma}}=\operatorname{Re} \sqrt{\frac{d \kappa}{2}}=\operatorname{Re} \sqrt{\frac{d}{2 R_{H}\left[1+\left(\frac{p}{2 \pi R_{H}}\right)^{2}\right]}}
$$

where $\gamma, \kappa$ and $p$ are respectively the radius of curvature, the curvature (i.e. the inverse of $\gamma$ ) and the pitch of the helix. Unlike Eq.2, this expression for the Dean number is consistent with the original definition, Eq. 1. Indeed, in the case of a torus/bend, the radius, $R$, and the radius of curvature, $\gamma$, are identical. Comparing Equations 2 and 3 , it can be noticed that they lead to significantly different results in the case of geometries with a high pitch to helix radius ratio, as $\gamma$ becomes much higher than $R_{H}$. To the authors' best knowledge, among the studies dealing with friction factor in helical pipes (Table 1), only Mishra and Gupta (1979) have based their correlation on the Dean number expression given in Equation 3.

In order to clarify the reasons why the Dean number definition given in Eq. 3 is much more convenient than that of Eq. 2, Figures 2 and 3 should be considered. Figure 2 presents the limits of the so-called forbidden region, i.e. the zone in which it is not possible to design helical shapes because the consecutive turns of the helix would intersect/overlap one with/on another. The equation of this frontier has been determined by Przybył and Pierański (2001). It represents the set of geometric parameters describing closely packed helixes, i.e. which pitch cannot be further decreased. Helix designs at some points are also represented at Figure 2. They illustrate the fact that the helical pipe geometry tends toward that of a straight tube at three asymptotic limits: (1) when the dimensionless pitch $p^{*}=p / d$ tends to infinity. (2) when the dimensionless helix radius $R_{H}^{*}=R_{H} / d$ tends to infinity. (3) when the dimensionless helix radius $R_{H}^{*}$ tends to zero.

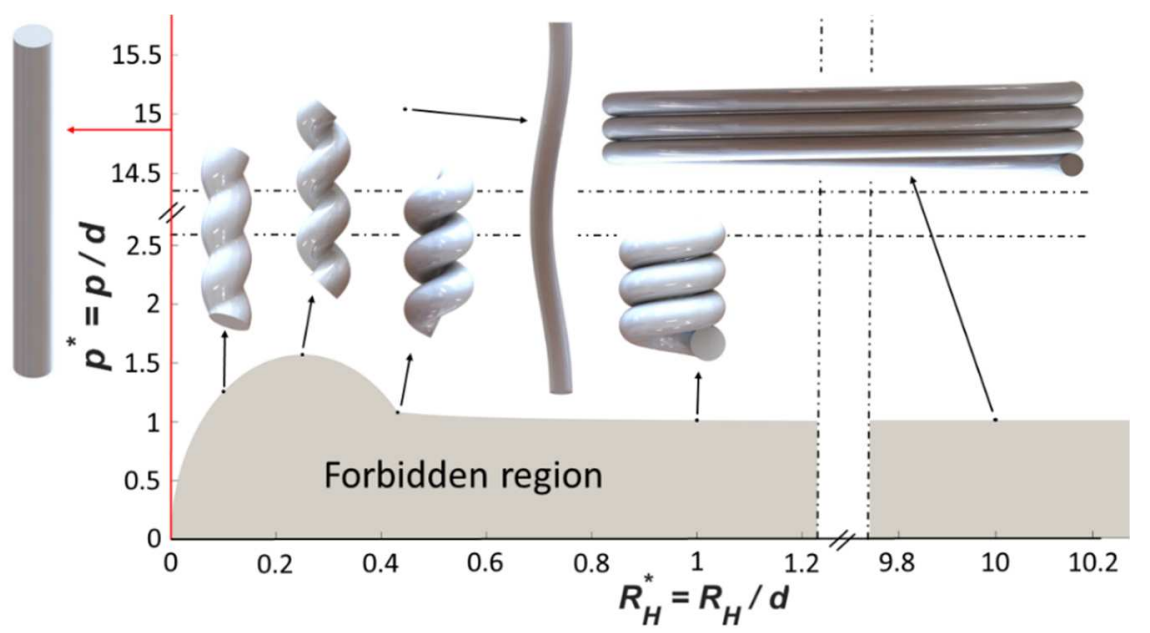

Figure 2: Limit of the forbidden region in the $\left(R_{H}^{*}, p^{*}\right)$ space (adapted from Przybył and Pierański (2001)) and some representative helix geometries.

Figure 3 shows the contour plot of the dimensionless helix curvature, $\kappa^{*}=\kappa \cdot d$, in the $\left(R_{H}^{*}, p^{*}\right)$ space. It illustrates the fact that $\kappa^{*}$ tends to zero at the abovementioned asymptotic limits, as the helix turns into a straight pipe. Thus, the Dean number as defined by Eq. 3 vanishes at these limits, which is consistent with the absence of Dean vortices and centrifugal effects in straight pipe flows. On the contrary, the Dean number as defined by Eq. 2 does not vanish at infinite helix pitches since it does not depend on the pitch value. More importantly, it tends 
to infinity when the helix radius approaches zero, which corresponds to Dean cells of infinite intensity. Therefore, as proved later in this paper, correlations based on this latter definition lead to systematic errors at low helix radiuses or relatively high helical pitches.

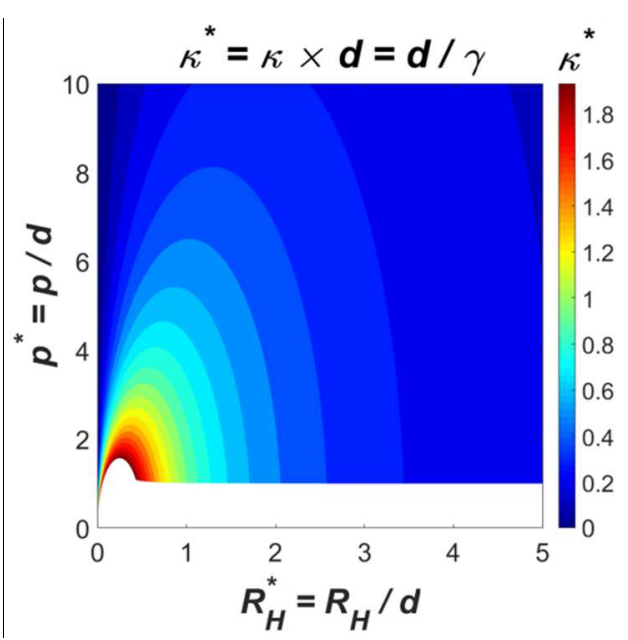

Figure 3: Contour plot of the dimensionless helix curvature in the $\left(R_{H}^{*}, p^{*}\right)$ space.

Moreover, Figure 3 reveals that the highest curvatures are associated to helixes of low pitch and relatively low helical radius. These geometries will be referred to as 'highly curved helical pipes' further on in this paper. They are expected to exhibit the highest centrifugal effects, i.e. the most intense Dean vortices. Indeed, a CFD (computational fluid dynamics) study has confirmed that the mass and/or heat transfer efficiencies in highly curved helical pipes can be an order of magnitude higher than in straight pipes, which makes these designs potentially interesting for heat exchangers and hollow fiber membranes applications (Abushammala et al., 2019). Moreover, their elongated shape allows them to be densely packed. Thus, highly curved helixes enable a great improvement of the overall transfer rates, and thus, allow a great unit volume reduction of heat/mass transfer equipment (Abushammala et al., 2019). It is worthy to underline that classic 'non-highly' curved helixes may lead to lower volumetric transfer rates than straight tubes. Indeed, the transfer efficiency improvement that they provide can be counterbalanced by their low packing density (Kaufhold et al. 2012).

Nevertheless, to the authors' knowledge, no other study has investigated yet the hydrodynamics or transport phenomena in highly curved helixes. As can be noticed from Table 1 for example, literature has focused on helixes with a high $R_{H}$ to $d$ ratio (i.e. $R_{H}^{*}$ ), probably because these geometries are easier to manufacture using conventional manufacturing techniques. However, nowadays, with the development of additive manufacturing, the elaboration of highly curved helical geometries has become easily achievable. It is noteworthy that 3D printing offers a great potential for improving heat exchangers and membranes designs (Cardone and Gargiulo, 2018; Low et al., 2017).

The current study focuses on laminar flow friction factor in highly curved helical pipes: laminar flow is encountered in many applications of practical interest, in particular small-scale devices, e.g. microfluidics, micro-structured heat exchangers and hollow fiber membranes. Although intense Dean vortices in highly curved helixes lead to improved heat and mass transfer efficiencies compared to straight pipes, they also induce higher pressure drops and hence greater pumping costs. Therefore, friction factor data in highly curved helixes are required so as to be able to compare the provided benefits with the additional operating costs and therefore to assess the usefulness of such designs.

In this paper, a series of CFD simulations are carried out to evaluate the friction factor in helical pipes, particularly highly curved ones. Different operating conditions, described by the Reynolds number, and various helix designs are examined. It is shown that available correlations fail in estimating the friction factor in highly curved helixes with a sufficient accuracy. Therefore, a new correlation predicting the friction factor over a wide range of helical pipe geometries and operating conditions is proposed. In order to validate the numerical results, a highly curved helical pipe was 3D-printed and pressure drops measurements are performed over it. The experimental data were in very good agreement with the proposed correlation results. Additionally, the correlation is shown to be robust, efficient and predictive since it correctly estimates experimental data from literature which were obtained under geometric and operating conditions beyond those investigated in the present paper (and upon which the correlation was built).

\section{Materials and methods}




\subsection{Friction factor and dimensional analysis}

In helical and curved pipes, the wall shear stress and so the local friction factor are not uniform over the pipe wall. In most engineering applications, only the friction coefficient averaged over the pipe circumference is of practical interest (it will be simply referred to as friction factor in the remaining part of this paper):

$$
C_{f}=\lim _{S \rightarrow 0}\left[\frac{8}{\rho U^{2}} \frac{1}{S} \iint \tau_{w} d S\right]
$$

150

where $C_{f}$ is the averaged local friction factor (defined after Darcy-Weisbach) at a given longitudinal position, $\tau_{w}$ the averaged local wall shear stress and $S$ the surface of a wall annular element sliced over the helix surface (Figure 4), and over which $\tau_{w}$ and $C_{f}$ are averaged.

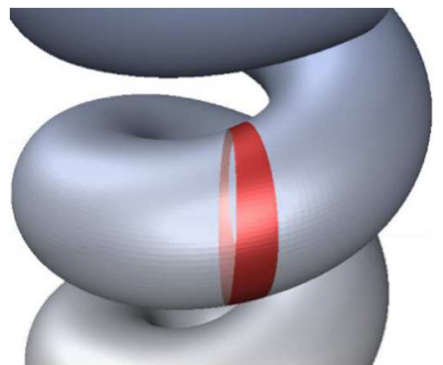

Figure 4: Shape of a wall element (in red) over which $C_{f}$ is averaged.

Figure 5 illustrates a qualitative variation of $C_{f}$ along a helical or a straight tube, the abscissa axis being the curvilinear - position along the pipe centerline. The friction factor is the highest at the pipe entrance and decreases over a relatively short distance called 'hydrodynamic entrance length' before reaching an asymptotic value, $C_{f}^{\infty}$. Even though $C_{f}^{\infty}$ is lower than the friction factor at the pipe entrance, whenever the pipe is sufficiently long, it gives the largest contribution to the mechanical energy dissipation, the so-called 'major head losses'. The additional head losses due to the higher friction coefficient at the entrance length are called 'minor losses'. The effects of minor losses and the hydrodynamic entrance length are generally negligible in the case of pipes of small diameter and/or sufficient length, which is generally the case in the applications targeted in this study, namely heat exchangers and hollow fiber membranes. Therefore, only major losses are addressed in this paper.

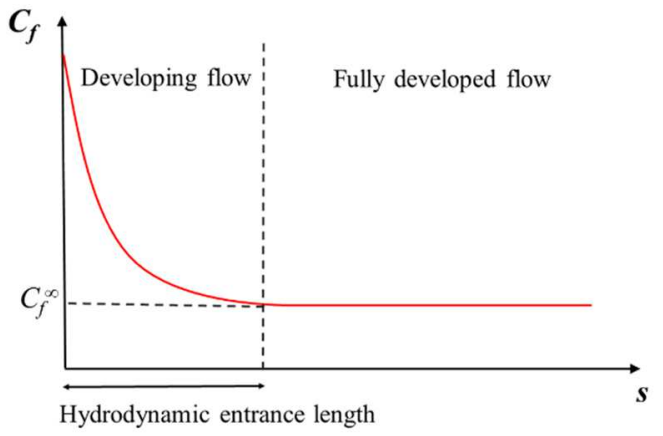

Figure 5: Typical variation of the friction factor from the entrance of a straight or a helical pipe.

Due to the occurrence of secondary flows, the asymptotic friction factor in helical pipes, $C_{f, H}^{\infty}$, is higher than that in straight ones, $C_{f, S}^{\infty}$ (which equals $64 / R e$ in the case of a laminar flow), under similar operating conditions. Assuming an incompressible flow under laminar conditions, $C_{f, H}^{\infty}$ value depends on the pipe internal diameter, $d$, the helical radius of the helix, $R_{H}$, its pitch, $p$, the fluid density, $\rho$, its dynamic viscosity, $\mu$, and the mean velocity of the primary flow, $U$. Therefore, according to the Buckingham $\pi$ theorem, the following three independent dimensionless parameters are required to correlate $C_{f, H}^{\infty}$ data: (1) The dimensionless helix pitch, $p^{*}=p / d$. (2) The dimensionless helix radius, $R_{H}^{*}=R_{H} / d$. These first two parameters characterize the helix shape. (3) The Reynolds number, $R e=\rho U d / \mu$, which accounts for the operating conditions.

\subsection{CFD modeling and simulation}


Flow simulations were conducted for various helical pipe designs and operating conditions. The geometries were drawn using Autodesk Inventor Professional 2018 software, considering a sufficient tube length to attain the fully developed flow region. The 3D numerical domain was meshed using ANSYS Meshing. The grid consisted of hexahedral cells only, with a boundary layer mesh in the near-wall zone for a more accurate calculation of the steep gradients prevailing in this zone. Based on preliminary simulations, the cells' size and density were chosen so at to ensure a mesh-independent solution.

CFD simulations were conducted using the commercial code ANSYS Fluent 16. The flow field in the pipe was determined by solving the continuity and Navier-Stokes equations in the case of a Newtonian, incompressible, isothermal and steady flow:

$$
\begin{gathered}
\operatorname{div}(\overrightarrow{\boldsymbol{v}})=0 \\
\rho \overrightarrow{\boldsymbol{d} \boldsymbol{v}}(\overrightarrow{\boldsymbol{v}} \otimes \overrightarrow{\boldsymbol{v}})=-\vec{\nabla} p+\mu \overrightarrow{\Delta \overrightarrow{\boldsymbol{v}}}
\end{gathered}
$$

Eqs. 5

where $\vec{v}$ is the velocity vector. As boundary conditions, a uniform velocity profile was set at the pipe inlet: as the flow rate is imposed, the gravity force has no effect on the velocity field and therefore this body force term was not included in the Navier-Stokes equations. It is noteworthy that the velocity profile at the inlet only affects the flow in the entrance length region and does not have any effect on the value of $C_{f, H}^{\infty}$. At the pipe outlet, a uniform pressure condition was used and the no-slip condition was set at the pipe walls. The advective terms in Eqs. 5 were discretized using the QUICK scheme while the diffusive ones were central-differenced. Pressure interpolation was carried out using a second order scheme. The equations were solved using a coupled iterative solver until convergence.

More than 150 simulations were carried out in the following range of operating conditions and aspect ratios: (a) $1.25 \leq p^{*} \leq 25$. (b) $0.05 \leq R_{H}^{*} \leq 10$. (c) $10 \leq R e \leq 2000$. In each simulation, the local friction factor was calculated at different longitudinal positions across the pipe length using Eq. 4 (considering a sufficiently small wall element as that depicted on Figure 4), and its asymptotic value, $C_{f, H}^{\infty}$, was determined. These CFD results were used to correlate $C_{f, H}^{\infty}$ as a function of the helix design and flow conditions. They are provided in an Excel sheet as supplementary material to this paper.

\subsection{D-printed helical pipe}

In order to validate the numerical results, a highly curved helical pipe was built with nylon material using a fused-filament 3D printer (Stream 30 Dual MK2). The geometric parameters of the helix that were targeted are summarized in the first column of Table 2.

Table 2: Targeted and obtained geometric parameters of the 3D-printed helical pipe.

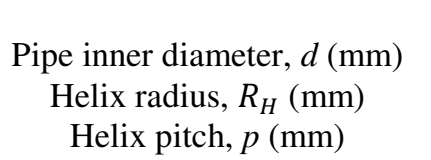

$\begin{array}{cc}\text { Targeted dimensions } & \text { Obtained dimensions } \\ 1.6 & 1.25 \pm 0.1 \\ 0.88 & 0.80 \pm 0.1 \\ 6 & 5.80 \pm 0.1\end{array}$

A first helical pipe having a wall thickness around $0.8 \mathrm{~mm}$ and a total height of $156 \mathrm{~mm}$ was 3D-printed (Figure 6a). Printing was carried out in the vertical direction, $z$. However, once the pipe has reached a certain height, the back and forth movement of the printing platform (in the $x$-direction) causes the pipe free end to vibrate: hence, the printing quality decreases and the pipe cross-section becomes no longer properly circular (Figure 6a). 

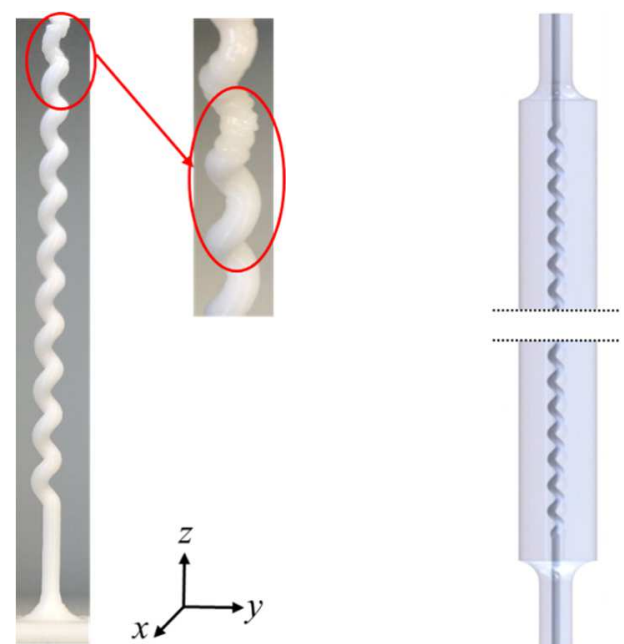

Therefore, a new design was tested (Figure 6b). It consists in surrounding the helical tube with a cylindrical block having an external diameter of $15 \mathrm{~mm}$. Indeed, weighting down the structure enhances its stability and reduces its oscillations during the printing process. This design is $200 \mathrm{~mm}$ high, which is the maximum height authorized by the 3D printer. It consists of a $156 \mathrm{~mm}$ high helical pipe connected at its ends to two straight pipes of $22 \mathrm{~mm}$ length each. The straight tubes are required to connect the pipe ends to a pressure transducer for pressure drop measurements.

The model represented in Figure 6b could be successfully 3D-printed without any detectable vibration to the naked eye. In order to check the printing quality, the pipe interior has been visualized using magnetic resonance imaging (MRI), a non-intrusive imaging technique commonly used in medical investigations. The pipe was filled with water and plugged at both ends and then introduced into the scanner. The model was visualized at different sections with different scanning angles. MRI images showed that the helical tube cross-section was properly circular and uniform all over the pipe length. However, it revealed that the tube internal diameter is slightly lower than the one initially targeted (Table 2). This difference is mainly due to the limited precision of the 3Dprinter (especially in the $x$ and $y$ directions) and to the relatively large diameter of the nozzle used, which is about $0.4 \mathrm{~mm}$.

The geometric parameters of the obtained helical pipe are summarized in the second column of Table 2: they correspond to a $R_{H}^{*}$ of about 0.64 and a $p^{*}$ around 4.64. The total curvilinear length of the tube is about $206 \mathrm{~mm}$, and is calculated as follows:

$$
L=n\left[\left(2 \pi R_{H}\right)^{2}+p^{2}\right]
$$

where $n$ is the number of helix turns which is equal to the total height of the helical pipe divided by its pitch.

\subsection{Experimental setup for pressure drop measurements}

The experimental setup for pressure drop measurements is represented in Figure 7. The 3D-printed model was placed horizontally and fed with an aqueous glycerol solution using a high precision micro annular gear pump (MZR-7205, HNP mikrosysteme, Schwerin, Germany. Operating flow rate range: 0.048-288 ml/min). A differential pressure sensor (Kobold PAD-d, Kobold instruments, Pittsburgh, USA. Operating range from 19 mbar to 1.9 bar, with a precision of about 2 mbar) connected to both ends of the pipe (Figure 6b) allows measuring the total pressure drop across the system. Three aqueous solutions with different glycerol concentrations were used as working fluids. Their dynamic viscosity was measured and their Newtonian behavior checked using a strain-imposed rheometer (AREF, TA Instrument, New Castle, USA) equipped with a parallel plates geometry of $50 \mathrm{~mm}$ diameter. To ensure reliable viscosity measurements, only solutions of viscosity higher than 20 times the viscosity of water were used.

Steady-state pressure drop measurements were conducted for twelve different Reynolds numbers ranging from 10 to 65 . Three replicates were performed for each measurement. The investigated operating conditions were chosen so as to respect the precision limits and the optimal working ranges of the pump, pressure transducer and rheometer. They are summarized in an Excel file provided as supplementary material to this paper. To the authors' best knowledge, these data constitute the first pressure drop measurements in highly curved helical pipes. 


\section{Results and discussion}

\subsection{CFD results}

Figure 8 shows contour plots of the $C_{f, H}^{\infty}$ to $C_{f, S}^{\infty}$ ratio that were obtained using a triangulation-based cubic interpolation of the CFD results. The geometric parameters for which simulations were performed are represented by black dots. It is worthy to note that, at these points, the values of $C_{f, H}^{\infty}$ (divided by $C_{f, S}^{\infty}$ ) that are displayed on the contour plots are exactly the same than those provided by CFD. On the other hand, the results predicted between these points are generated by interpolation. Therefore, they may be expected not to be very accurate since they are quite sensitive to the interpolation scheme used. Indeed, as can be noticed from Figure 8, the contour plots exhibit some relatively irregular variations, and at some positions, the predicted $C_{f, H}^{\infty}$ to $C_{f, S}^{\infty}$ values are even slightly lower than unity.

(a)

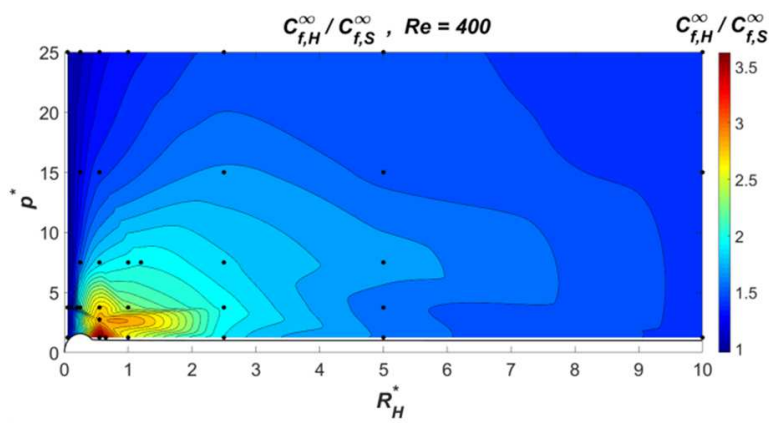

(b)

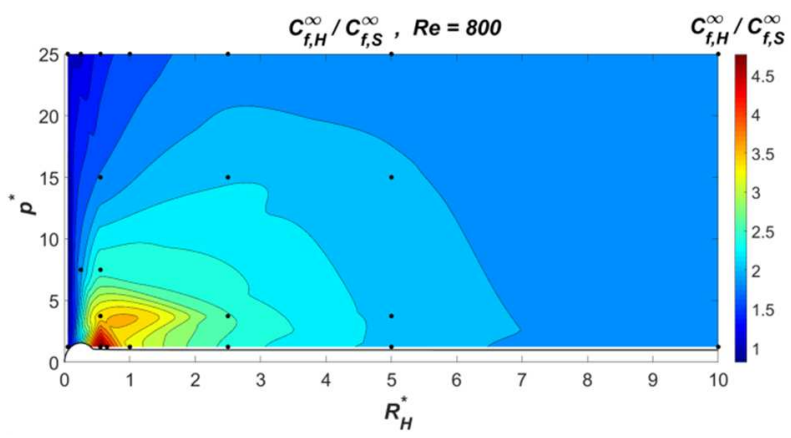

Figure 8: Contour plots of the $C_{f, H}^{\infty}$ to $C_{f, S}^{\infty}$ ratio at a Reynolds number of (a) 400 and (b) 800 respectively. The black dots represent the geometric conditions for which CFD results are acquired $\left(1.25 \leq p^{*} \leq 25\right.$ and $\left.0.05 \leq R_{H}^{*} \leq 10\right)$. The contours are derived by interpolating these data.

Nonetheless, these contour plots provide valuable information:

(1) Comparison to Figure 3 confirms that the more curved is the pipe, the higher the magnitude of $C_{f, H}^{\infty}$. Indeed, highly curved helixes engender the most intense Dean-type vortices and hence the highest friction factors.

(2) For a given dimensionless pitch, $p^{*}$, there exists a dimensionless helix radius, $R_{H}^{*}$, at which the friction coefficient is maximal. The $C_{f, H}^{\infty}$ to $C_{f, S}^{\infty}$ ratio decreases for higher or lower helical radiuses and tends toward unity when $R_{H}^{*}$ tends to zero or to infinity as the helix geometry approaches that of a straight pipe.

(3) For a given $R_{H}^{*}$, the $C_{f, H}^{\infty}$ to $C_{f, S}^{\infty}$ ratio decreases when $p^{*}$ increases (although interpolated data indicate the opposite at some positions). Indeed, as can be noticed from Figure 3 and Eq. 3, the pipe curvature (and hence centrifugal effects) diminishes when $p^{*}$ is increased. In fact, the $C_{f, H}^{\infty}$ to $C_{f, S}^{\infty}$ ratio should tend towards unity at infinite $p^{*}$ as the helix design approaches a straight pipe.

(4) Although $C_{f, H}^{\infty}$ decreases when $p^{*}$ is increased, as can be seen from Figure 8 , this effect is only significant in the case of highly curved helixes. Indeed, for relatively high $R_{H}^{*}$, the curvature is not much sensitive to the value of $p^{*}$ (Figure 3). As only helical pipes with rather large $R_{H}^{*}$ were investigated in the literature, most authors concluded that the pitch value has no significant effect on $C_{f, H}^{\infty}$ (Table 1). Thus, it is obvious that their 
correlations, which are based on $D e_{R_{H}}$ (Eq. 2), cannot accurately predict the friction coefficient in highly curved helical pipes. Only the one derived by Mishra and Gupta (1979) may lead to acceptable results since it considers pitch effects through the use of $D e_{\gamma}$ (Eq.3).

(5) When $R e$ is increased, centrifugal effects get more intense, thus the flow and so $C_{f, H}^{\infty}$ become more sensitive to the helix geometry. Hence, while the maximum value of the $C_{f, H}^{\infty}$ to $C_{f, S}^{\infty}$ ratio is about 3.5 under $R e=400$, it exceeds 4.5 for $R e=800$. However, the geometric parameters for which $C_{f, H}^{\infty}$ is maximal do not seem to be significantly affected by the Reynolds number value.

\subsection{Correlation development}

Although the $C_{f, H}^{\infty}$ field in the $\left(R_{H}^{*}, p^{*}\right)$ space (Figure 8) presents a single maximum (no local optima) for a given $R e$, finding a mathematical model that correctly fits the CFD data was very challenging and tedious. In fact, the $C_{f, H}^{\infty}$ field exhibits steep variations in the highly curved helixes region, with highly non-uniform and anisotropic gradients, which magnitudes are very sensitive to the helix geometry.

Accordingly, the development of the regression model for $C_{f, H}^{\infty}$ has been conducted by trial-and-error. The following expression has been found to provide the best fit of the CFD data:

where:

$$
\begin{gathered}
C_{f, H}^{\infty}=\frac{64}{R e}+A \cdot B \cdot \exp (-C) \\
A=p_{1} \cdot D \cdot\left(\frac{D}{R e}\right)^{p_{2}} \\
B=\left(R_{H}^{*}+\frac{1}{R_{H}^{*}}\right)^{p_{3}} \\
C=p_{4} \cdot D \cdot p^{*} \cdot R_{H}^{*}-p_{5} \\
D=\left[R_{H}^{*} p_{6}\left(1+\left(\frac{p^{*}}{2 \pi R_{H}^{*}}\right)^{2}\right)\right]^{-p_{7}}
\end{gathered}
$$

The present expression includes 7 positive regression parameters denoted $p_{i}(i$ being an integer ranging from 1 to 7) which values are determined using an optimization procedure, as explained later, in order to minimize the differences between the correlation outputs and the CFD data.

The term $64 / R e$ corresponds to the asymptotic friction factor in a straight tube, $C_{f, S}^{\infty}$, with the DarcyWeisbach definition. $A$ and $B$ being positive terms, for any given $R e$, the present correlation guarantees that the predicted $C_{f, H}^{\infty}$ value remains greater (or equal) than $C_{f, S}^{\infty}$.

Coefficient $D$ is analogous to $\kappa^{*}$, with the difference that $p_{6}$ and $p_{7}$ are treated as optimization variables instead of assigning their values to 1 and 2 respectively. Parameters $p_{6}$ and $p_{7}$ being strictly positive, term $D$ vanishes when $R_{H}^{*}$ tends to zero or infinity or when $p^{*}$ tends to infinity, i.e. when the helical pipe geometry tends toward that of a straight one.

The structure of term $A$ expresses the fact that the magnitude of $C_{f, H}^{\infty}$ increases in zones of high curvatures and decreases with increasing $R e$ (as the optimal value of $p_{2}$ was found to be strictly positive). The term $D$ appears with a strictly positive power, $1+p_{2}$, in the expression of $A$. Accordingly, term $A$ vanishes at the asymptotic limits for which the helical pipe reduces to a straight one. Thus, the structure of the developed correlation guarantees that the predicted $C_{f, H}^{\infty}$ becomes equal to $C_{f, S}^{\infty}$ at these limits.

Term $B$ allows adjusting the model behavior at large and low $R_{H}^{*}$ values, and its incorporation into the mathematical expression significantly improved the correlation accuracy.

Term $C$ being positive, $\exp (-C)$ represents a decreasing exponential. Since $D$ appears with a positive power in the expression of $C, \exp (-C)$ allows reproducing the steep variations of $C_{f, H}^{\infty}$ in the regions of high helix curvature, and the flattening of the $C_{f, H}^{\infty}$ contour plot in zones of low helix curvature, namely at high $R_{H}^{*}$ and $p^{*}$ values.

The mathematical expression presented in Equations 7 was used to correlate the CFD data. It should be recalled that, as mentioned in Section 2.2, these results were obtained in the following range of dimensionless parameters: $1.25 \leq p^{*} \leq 25,0.05 \leq R_{H}^{*} \leq 10,10 \leq R e \leq 2000$. For a better accuracy, two correlations were derived: the first one using the data where $R e$ ranges from 10 to 400, and the second one for $R e$ ranging from 400 to 2000 . The optimization problem was formulated as a minimization of the maximum relative difference between the model predictions and the numerical data. The optimal set of the parameters $p_{i}$, which is reported in 
Table 3, was determined using a genetic algorithm and the results were further refined using a local optimizer, GRG2.

Table 3: Optimal set for the parameters of the correlation expressed by Equations 7.

Validity range
$10 \leq R e \leq 400$
$400 \leq R e \leq 2000$

$\begin{array}{cc}\boldsymbol{p}_{\boldsymbol{1}} & \boldsymbol{p}_{\boldsymbol{2}} \\ 1.98 & 4.07 \times 10^{-1} \\ 2.88 & 3.82 \times 10^{-1}\end{array}$

$p_{3}$
$8.49 \times 10^{-1}$

$9.16 \times 10^{-3}$

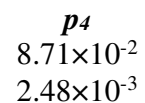

$\begin{array}{cc}p_{6} & p_{7} \\ 2.31 & 3.67 \times 10^{-1} \\ 1.10 & 3.23 \times 10^{-1}\end{array}$

Figure 9 compares the correlation predictions and CFD results (represented by brown disks). It shows that the proposed correlation correctly fits the $C_{f, H}^{\infty}$ data as most points are within an error margin of $10 \%$. Indeed, the maximum relative difference between the numerical results and the correlation predictions is respectively $13.8 \%$ for $R e$ below and 400 and $13.2 \%$ for $R e$ above 400 .

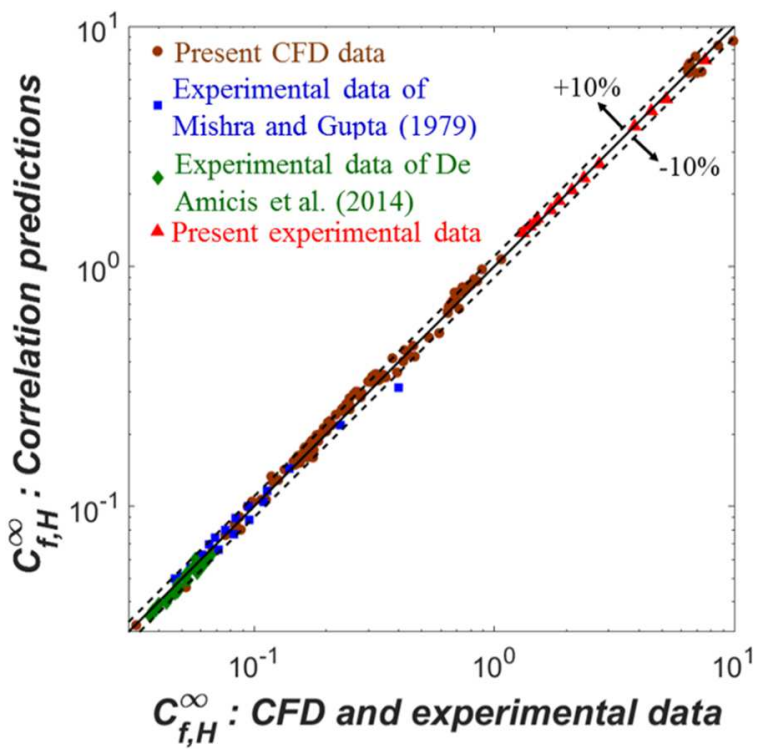

Figure 9: Parity diagram of $C_{f, H}^{\infty}$ : CFD and experimental data versus correlation predictions.

Figure 10 shows the contour plot of the $C_{f, H}^{\infty}$ to $C_{f, S}^{\infty}$ ratio calculated using the present correlation for a $R e$ of 800. Comparison to Figure $8 \mathrm{~b}$ shows a good agreement, with the difference that the model data are much smoother since they are not affected by interpolation approximations. As can be noticed from Figure $10, C_{f, H}^{\infty}$ exhibits the largest values as well as the highest gradients in the highly curved helixes region. The $C_{f, H}^{\infty}$ to $C_{f, S}^{\infty}$ ratio tends toward unity at very low values of $R_{H}^{*}$. Indeed, the formulation of the mathematical expression (Eqs. 7) ensures that $C_{f, H}^{\infty}$ becomes equal to $C_{f, S}^{\infty}$ at the three asymptotic limits at which the helix geometry tends toward that of a straight pipe.

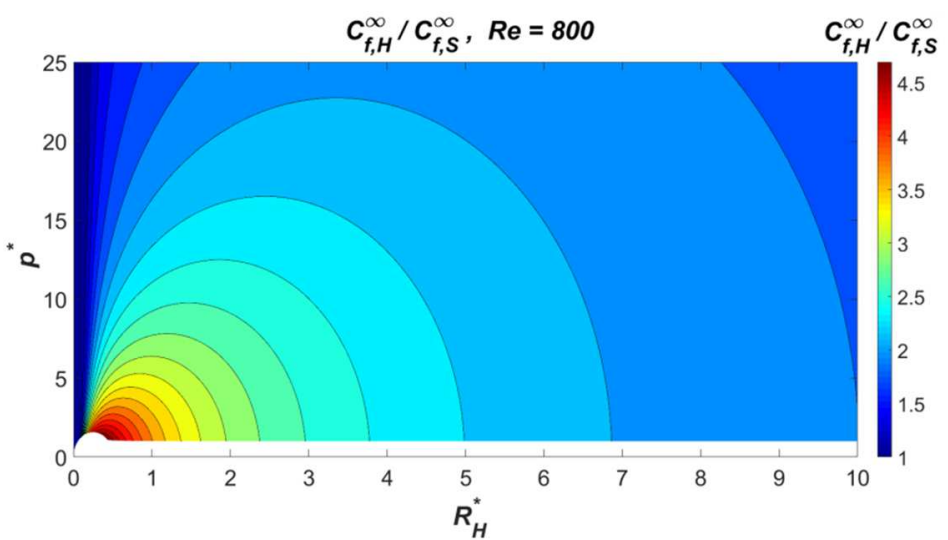

Figure 10: Contour plot of the $C_{f, H}^{\infty}$ to $C_{f, S}^{\infty}$ ratio at a Reynolds number of 800: present correlation data (Equations 7).

\subsection{Comparison with literature correlations}


In order to assess the potentiality of the new correlation, its performance are compared to that of literature correlations in Figures 11, 12 and 13. Figure 11 depicts the variations of $C_{f, H}^{\infty}$ as a function of $R_{H}^{*}$ for two values of $p^{*}$. Given that literature correlations were derived by regressing experimental measurements acquired on helixes with large $R_{H}^{*}$ (Table 1), they are expected to be accurate for non-highly curved helixes. As can be noticed, at relatively large $R_{H}^{*}$ values, the results provided by all of the plotted correlations are in good agreement with the current CFD data. This constitutes a first validation of the present CFD simulations.
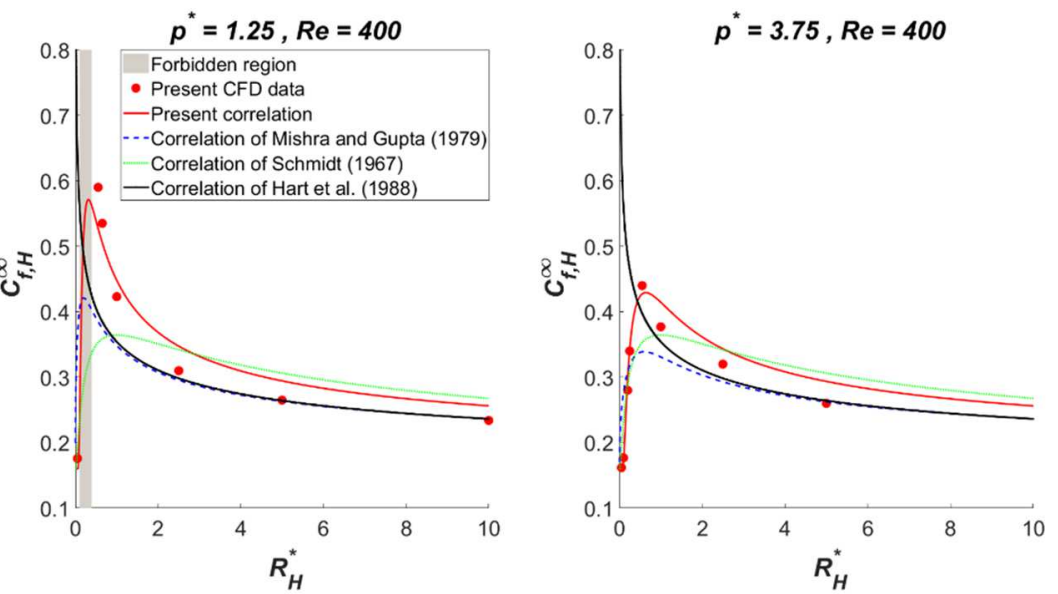

Figure 11: $C_{f, H}^{\infty}$ versus $R_{H}^{*}$ at two different $p^{*}$ : comparison of different correlations.

However, Figure 11 also shows that literature correlations do not correctly estimate $C_{f, H}^{\infty}$ in the case of highly curved helixes. Indeed, the correlation of Hart et al. (1988) and all the correlations based on $D e_{R_{H}}$ (Equation 2) (see Table 1) do predict an infinite friction factor when $R_{H}^{*}$ tends towards zero.

On the other hand, the correlations of Schmidt (1967) and Mishra and Gupta (1979) reproduce correctly the shape of the curves showing $C_{f, H}^{\infty}$ versus $R_{H}^{*}$. However, the correlation of Schmidt (1967) does not account for any pitch effect. Therefore, it leads to systematic errors in the highly curved helixes zone where the friction factor is very sensitive to the pitch (Figures 8, 10, 11 and 12). The correlation of Mishra and Gupta (1979) proved to be the most accurate among literature models. In some cases as illustrated in Figure 11, it does even provide slightly better predictions that the current correlation. However, its major weak point is that it generally largely underestimates the friction factor in highly curved helixes as can be noticed from Figures 11, 12 and 13.
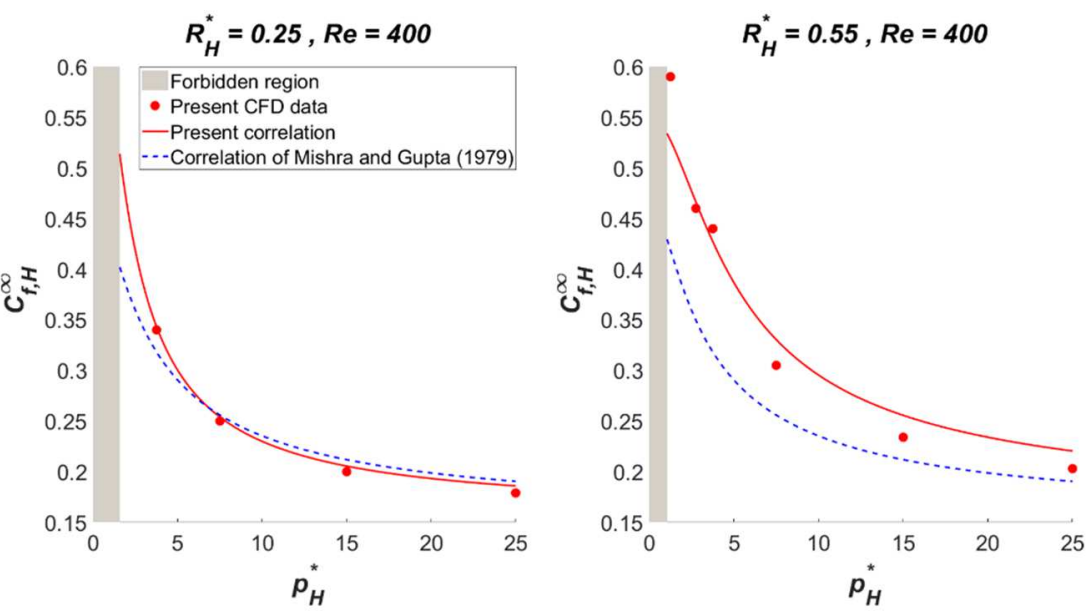

Figure 12: $C_{f, H}^{\infty}$ versus $p^{*}$ at two different $R_{H}^{*}$ : comparison between the present correlation and that of Mishra and Gupta (1979).

Finally, the variations of $C_{f, H}^{\infty}$ with the Reynolds number are plotted on Figure 13 for four highly curved helixes. This figure illustrates the fact that the present model (Eqs. 7) correctly captures the Re effects, and is generally more accurate than that of Mishra and Gupta (1979). A discontinuity in the curves obtained with the present correlation can be noticed. It is due to the use of different parameter values depending on whether $R e$ is below or above 400 (Table 3). 

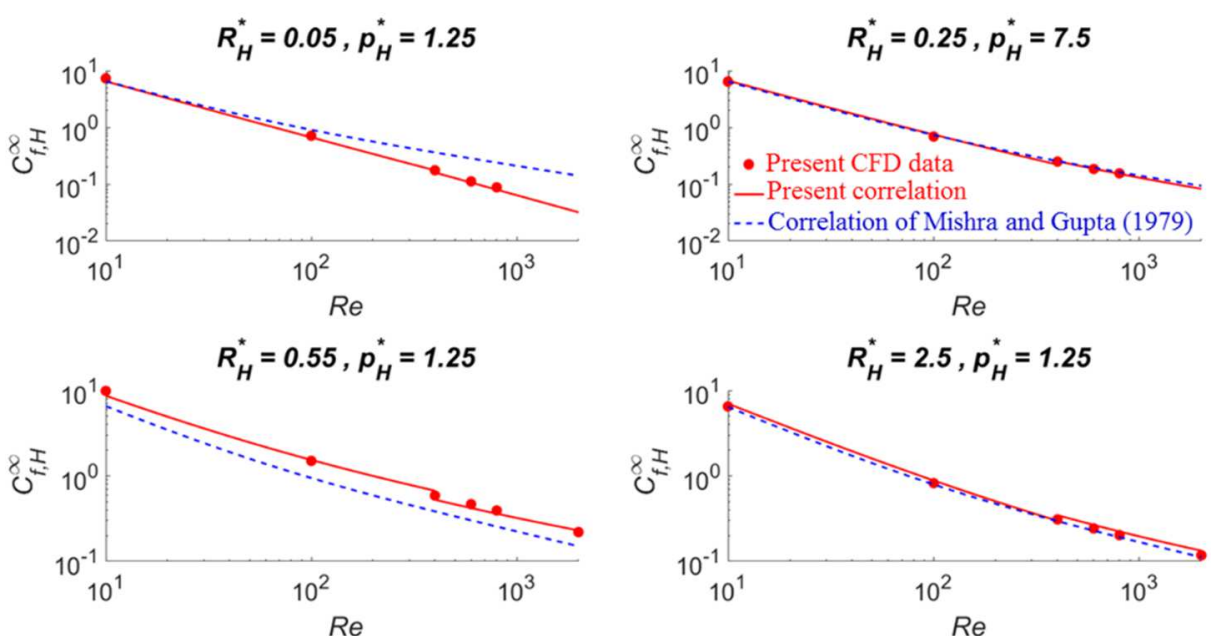

Figure 13: $C_{f, H}^{\infty}$ variation with $R e$ for four different helixes.

\subsection{Correlation validation using experimental data from literature}

In addition to CFD data, for a more trustworthy validation, the correlation results were compared to the experimental data of Mishra and Gupta (1979) and De Amicis et al. (2014). Table 4 summarizes the geometric and operating conditions over which these measurements were obtained. It is noteworthy that, although most of these experiments were performed for a $R e$ higher than 2 300, they do all correspond to a laminar flow regime. Indeed, in the case of helical pipes, centrifugal forces do generally have a stabilizing effect on the flow which delays the transition to turbulence. Hence, the flow can remain laminar for Reynolds values much higher than in straight pipes (De Amicis et al., 2014; Ghobadi and Muzychka 2016).

The last column in Table 4 recalls the range of geometric and operating conditions over which the current CFD data were performed, and hence, upon which the present correlation was built. It can be noticed that all of the literature experimental results were acquired for conditions beyond the validity range of the present correlation. Nevertheless, as shown in Figures 9 and 14, all of De Amicis et al. (2014) results are predicted within a margin error of $10 \%$, and most of Mishra and Gupta (1979) data are predicted with a relative error less than $8 \%$. Only a single point falls beyond this limit and is predicted with an error of about $28 \%$. It corresponds to a measurement acquired by Mishra and Gupta (1979) under a $R e$ of 266, which represents the lowest $R e$ investigated in their study (Table 4). Such a low Re leads to a low pressure drop especially since the helix considered is not highly curved; hence, this measurement is expected to be much affected by experimental errors.

Table 4: Ranges of geometric and operating conditions over which the literature experimental data were acquired and the present correlation was built.

$\begin{array}{ccccc} & \begin{array}{c}\text { Mishra and Gupta } \\ (\text { 1979): helical pipe 1 }\end{array} & \begin{array}{c}\text { Mishra and Gupta } \\ (1979): \text { helical pipe 2 }\end{array} & \begin{array}{c}\text { De Amicis et al. } \\ (2014)\end{array} & \begin{array}{c}\text { Range of parameters investigated } \\ \text { using CFD and upon which the } \\ \text { present correlation was built }\end{array} \\ R_{H}^{*} & 3.4 & 20.2 & 39.9 & {[0.05-10]} \\ p^{*} & 27.5 & 18.9 & 63.8 & {[1.25-25]} \\ R e & {[266-5500]} & {[1100-2550]} & {[1790-4160]} & {[10-2000]}\end{array}$

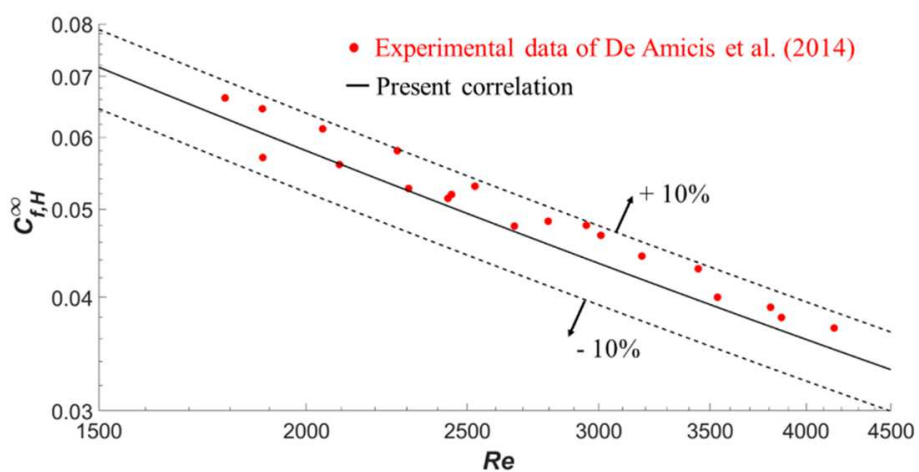


The results presented in this section demonstrate the predictive capacities of the present correlation, as it successfully estimated experimental data which were acquired beyond its supposed range of validity. This advantageous feature is probably due to the structure of the present model (Eqs. 7): in particular, it guarantees correct results at the asymptotic limits where the helical pipe geometry tends toward that of a straight one, i.e. it ensures results to be correctly bounded. Therefore, the present correlation is believed to lead to realistic and accurate friction factor values even beyond the range of geometric and operating conditions investigated in this paper.

\subsection{Correlation validation using data acquired on the 3D-printed highly curved helix}

The performed pressure measurements correspond to the total pressure drop across the 3D-printed model, i.e. through the helical pipe (which $R_{H}^{*}$ is about 0.64 and $p^{*}$ around 4.64, see Section 2.3) and the two straight pipes connections. Assuming that the friction factor in the straight tubes equals 64/Re, the pressure drop across the helical pipe, $\Delta p_{H}$, could be calculated (calculation details are given in the Excel file provided as supplementary material to this paper). Given the important length and the small diameter of the helical pipe, minor head losses can be neglected. Thus, $C_{f, H}^{\infty}$ is calculated following Darcy-Weisbach:

$$
C_{f, H}^{\infty}=\frac{\Delta p_{H} d}{\frac{1}{2} \rho L U^{2}}
$$

where $L$ is the total curvilinear length of the helical pipe.

Figures 9 and 15 compare the experimental friction factor to the correlation (Equations 7) predictions. All the experimental data are predicted within a relative error margin less than $8 \%$, which demonstrates the accuracy of the proposed correlation in the case of highly curved helical pipes.

This correlation is the first one to accurately predict the pressure drop in highly curved helical pipes. Moreover, as shown in this paper, it proved to be efficient over a broad range of geometric and operating conditions. The precise estimation of the specific energy requirement of a whole set of heat and mass exchangers designs, over a broad range of operating conditions (under the assumption of a laminar flow regime) is thus now achievable. This offers the possibility to systematically explore which heat or mass exchanger geometry offers the best combination of increased transfer performances together with a minimal specific energy requirement. Process intensification studies typically correspond to such an objective and the results reported in this paper should be helpful for optimal exchangers design studies.

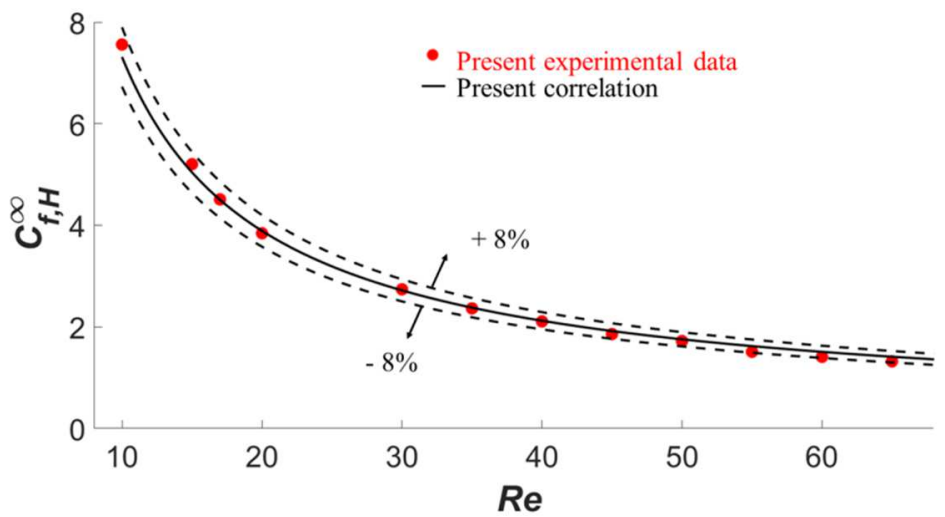

Figure 15: Comparison between the present correlation predictions and the present experimental data.

\section{Conclusion and perspectives}

This paper focused on the laminar flow friction factor in helical pipes, especially highly curved geometries. CFD simulations were conducted to determine the friction factor for various helix designs under different Reynolds numbers. On the basis of these numerical results, a general correlation for calculating the friction factor in helical pipes was developed. The correlation proved to be robust, efficient and predictive as its results were in excellent agreement with literature experimental data as well as with measurements performed on a 3Dprinted highly curved helical tube. 
Future studies will concentrate on developing correlations predicting the heat and mass transfer efficiencies in helical pipes. These correlations, along with the one developed in this paper, will then be used in model-based optimization of helically coiled heat exchangers and hollow fiber membrane contactors in order to determine the optimal helical tubes design, i.e. the one leading to the most lucrative trade-off between the transfer efficiency enhancement and the increase of pumping costs.

\section{Acknowledgment}

The authors gratefully thank the 'French ministry of higher education and research' for funding this study, Mrs. Maude Ferrari for the MRI measurements and Mr. Philippe Marchal for the rheological experiments.

\section{References}

Abdel-Aziz M. H., Mansour I. A. S., Sedahmed, G. H., 2010. Study of the rate of liquid-solid mass transfer controlled processes in helical tubes under turbulent flow conditions. Chemical Engineering and Processing: Process Intensification, 49(7), 643-648.

Abushammala O., Hreiz R., Lemaître C., Favre E., 2019. Maximizing mass transfer using highly curved helical pipes: A CFD investigation. $6^{\text {th }}$ International Conference on Fluid Flow, Heat and Mass Transfer (FFHMT'19), June 18 - 19, 2019 Ottawa, Canada.

Ali S., 2001. Pressure drop correlations for flow through regular helical coil tubes. Fluid Dynamics Research, 28(4), 295.

Cardone M., Gargiulo B., 2018. Design and experimental testing of a Mini Channel Heat Exchanger made in Additive Manufacturing. Energy Procedia, 148, 932-939.

De Amicis J., Cammi A., Colombo L. P., Colombo M., Ricotti M. E., 2014. Experimental and numerical study of the laminar flow in helically coiled pipes. Progress in Nuclear Energy, 76, 206-215.

Dean, W. R., 1927. Note on the motion of fluid in a curved pipe. Philosophical Magazine and Journal of Science, 4(20), 208223.

Dean, W. R., 1928. The stream-line motion of fluid in a curved pipe. Philosophical Magazine and Journal of Science, 5(30), 673-695

Ghidossi R., Veyret D., Moulin, P., 2006. Computational fluid dynamics applied to membranes: State of the art and opportunities. Chemical Engineering and Processing: Process Intensification, 45(6), 437-454.

Gill J., Singh J., 2018. Use of artificial neural network approach for depicting mass flow rate of R134a/LPG refrigerant through straight and helical coiled adiabatic capillary tubes of vapor compression refrigeration system. International Journal of Refrigeration, 86, 228-238.

Ghobadi M., Muzychka Y. S., 2016. A review of heat transfer and pressure drop correlations for laminar flow in curved circular ducts. Heat Transfer Engineering, 37(10), 815-839.

Hart J., Ellenberger J., Hamersma P. J., 1988. Single-and two-phase flow through helically coiled tubes. Chemical Engineering Science, 43(4), 775-783.

Kaufhold D., Kopf F., Wolff C., Beutel S., Hilterhaus L., Hoffmann M., Scheper T., Schlüter M., Liese A., 2012. Generation of Dean vortices and enhancement of oxygen transfer rates in membrane contactors for different hollow fiber geometries. Journal of membrane science, 423, 342-347.

Liu Y., Chen Y., Zhou Y., Wang D., Wang Y., Wang D., 2019. Experimental research on the thermal performance of PEX helical coil pipes for heating the biogas digester. Applied Thermal Engineering, 147, 167-176.

Low Z. X., Chua Y. T., Ray B. M., Mattia D., Metcalfe I. S., Patterson, D. A., 2017. Perspective on 3D printing of separation membranes and comparison to related unconventional fabrication techniques. Journal of Membrane Science, 523, 596613.

Mansour M., Liu Z., Janiga G., Nigam K. D., Sundmacher K., Thévenin D., Zähringer, K., 2017. Numerical study of liquidliquid mixing in helical pipes. Chemical Engineering Science, 172, 250-261.

Mendez D. L. M., Lemaitre C., Castel C., Ferrari M., Simonaire H., Favre E., 2017. Membrane contactors for process intensification of gas absorption into physical solvents: Impact of dean vortices. Journal of Membrane Science, 530, 2032.

Mishra P., Gupta S. N., 1979. Momentum transfer in curved pipes. 1. Newtonian fluids. Industrial \& Engineering Chemistry Process Design and Development, 18(1), 130-137.

Moll R., Veyret D., Charbit F., Moulin P., 2007. Dean vortices applied to membrane process: Part I. Experimental approach. Journal of Membrane Science, 288(1-2), 307-320.

Mori Y., Nakayama W., 1965. Study of forced convective heat transfer in curved pipes. International journal of heat and mass transfer, 8, 67-82.

Przybył S., Pierański P., 2001. Helical close packings of ideal ropes. The European Physical Journal E, 4(4), 445-449.

Pioro I. (Editor), 2016. Handbook of generation IV nuclear reactors. Woodhead Publishing.

Schmidt E. F., 1967. Wärmeübergang und druckverlust in rohrschlangen. Chemie Ingenieur Technik, 39(13), 781-789.

Srinivasan P.S., Nandapurkar S.S., Holland F.A., 1968. Pressure drop and heat transfer in coils. The Chem. Eng. (London), 218, CE113-119.

White C. M., 1929. Streamline flow through curved pipes. Proc. R. Soc. Lond. A, 123(792), 645-663. 